\title{
The peculiar physiology of the python
}

by Gregory D. Larsen

\section{SCIENTIFIC NAME}

Python bivittatus

TAXONOMY

PHYLUM: Chordata

CLASS: Reptilia

ORDER: Squamata

FAMILY: Pythonidae

\section{General description}

The Burmese python is one of the largest snakes in the world with an average length of about $3.7 \mathrm{~m}$, though they can range up to $5.74 \mathrm{~m}$ in length. These snakes are native to much of South and Southeast Asia, though in recent decades the exotic pet trade has distributed them around the world, and there is now a well-established invasive population in south Florida. In the wild, Burmese pythons are associated with forested and, often, aquatic habitats. They are primarily nocturnal ambushhunters, though there have also been many documented cases of diurnal and opportunistic foraging behaviors. Pythons kill by constriction, and adult Burmese pythons can consume prey up to 1.6 times their own body mass. Owing to their popularity as pets, there are many resources available from commercial breeders and hobbyists that detail the husbandry requirements of captive pythons, and published literature describes relatively simple practices for maintaining these snakes in laboratories for experimental research ${ }^{1-3}$.

\section{Research résumé}

As an ambush predator, Burmese pythons regularly go without feeding for long periods of time. When they do feed, however, pythons hunker down motionless, for days at a time to digest their meals, which are often very large and swallowed whole. What follows is an intense digestive process that is characterized by dramatic physiological changes, and for this process Burmese pythons have been proposed as a model of extreme adaptations, regulatory biology, digestive physiology and overall physiological plasticity ${ }^{4}$. Within days of feeding, a python's metabolic rate surges from its fasting levels to a rate that is comparable to that of a galloping horse; however, a python can sustain this level for as long as 16 days, if needed, to digest its meal ${ }^{5}$.

It is the rapidity with which these snakes shift from a stable, fasting state to a sustained digesting response that presents a promising model for researchers that seek to understand how regulatory systems mediate physiological changes in vertebrates. Within days of feeding, most of a python's major organs increase in size by $35-100 \%$ alongside drastic changes in blood chemistry; they then return to fasting sizes by the time of defecation ${ }^{6}$. It is especially interesting that many of these changes take place in the absence of cell proliferation. For example, to support the overall increase in metabolic rate, the heart undergoes massive cellular hypertrophy, adding $40 \%$ to its mass to produce a much greater cardiac output. This growth is accompanied by protective enzymes and physiological pathways that safeguard the heart from lipid deposition and oxidation. This entire process appears to be mediated by a mixture of fatty acids, which, when administered to mice, produced similar hypertrophy in their mammalian cardiomyocytes. This suggests that the system by which pythons regulate cardiac hypertrophy could be adapted to augment cardiac performance in human medicine ${ }^{7}$. To better understand this and the many other complex physiological responses of pythons dur-

ing feeding, a large team of researchers has sequenced and annotated the genome of the Burmese python and specifically studied the transcriptional responses of the heart, kidney, liver and small intestine after feeding ${ }^{8}$.

Although the Burmese python has achieved recognition as a nontraditional model of unique physiological adaptations, the majority of research with this species presently focuses on its significance as an invasive predator in the Everglades ecosystem. Laboratory experiments have already helped researchers refine predictive models of the unfolding invasion ${ }^{2}$ and test new toxicants as potential control agents ${ }^{3}$. As research with Burmese pythons develops in these multiple, disparate directions, researchers are bound to uncover still more unique characteristics in this unusual animal model.

1. Esbaugh, A.J., Secor, S.M. \& Grosell, M. Renal plasticity in response to feeding in the Burmese python, Python molurus bivittatus. Comp. Biochem. Physiol. A Mol. Integr. Physiol. 188, 120-126 (2015).

2. Hart, K.M., Schofield, P.J. \& Gregoire, D.R. Experimentally derived salinity tolerance of hatchling Burmese pythons (Python molurus bivittatus) from the Everglades, Florida (USA). J. Exp. Mar. Biol. Ecol. 413, 56-59 (2012).

3. Mauldin, R.E. \& Savarie, P.J. Acetaminophen as an oral toxicant for Nile monitor lizards (Varanus niloticus) and Burmese pythons (Python molurus bivittatus). Wildl. Res. 37, 215-222 (2010).

4. Carey, H.V. et al. Elucidating nature's solutions to heart, lung, and blood diseases and sleep disorders. Circ. Res. 110, 915-921 (2012).

5. Secor, S.M. \& Diamond, J. A vertebrate model of extreme physiological regulation. Nature 395, 659-662 (1998).

6. Secor, S.M. \& Diamond, J. Adaptive responses to feeding in Burmese pythons: pay before pumping. J. Exp. Biol. 198, 1313-1325 (1995).

7. Riquelme, C.A. et al. Fatty acids identified in the Burmese python promote beneficial cardiac growth. Science 334, 528-531 (2011).

8. Castoe, T.A. et al. The Burmese python genome reveals the molecular basis for extreme adaptation in snakes. Proc. Natl. Acad. Sci. USA 110, 2064520650 (2013). 